\title{
Preparation of Electron and X-Ray Transparent Inorganic Particles for Analytical Microscopy Using the Ultramicrotome
}

\author{
Mark Homer $^{1}$, Patrick Cappillino ${ }^{2}$, Farid El Gabaly ${ }^{1}$, Helmut Gnaegi ${ }^{3}$, Dave Robinson ${ }^{1}$, and Josh Sugar $^{1}$ \\ ${ }^{1}$ Sandia National Laboratories, Livermore, CA, USA. \\ ${ }^{2}$ University of Massachusetts Dartmouth, Chemistry and Biochemistry Department, North Dartmouth, \\ MA, USA. \\ ${ }^{3}$ Diatome Ltd., Biel-Bienne, Switzerland.
}

Many advanced materials characterization techniques that perform at the highest spatial and energy resolutions have strict dimensional requirements on acceptable samples. For example, the transmission electron microscope (TEM) generally requires samples to be electron transparent, which means samples must be $<100 \mathrm{~nm}$ thick and they must fit in a holder that typically holds $3 \mathrm{~mm}$-diameter discs. Electron energy loss spectroscopy (EELS) in the TEM may place further restrictions on sample thickness (e.g. < $50 \mathrm{~nm})$. Likewise, scanning transmission x-ray microscopy (STXM) requires samples be X-ray transparent for $\mathrm{x}$-ray absorption spectroscopy (XAS) and typically $<500 \mathrm{~nm}$ thick. Common sample preparation and thinning techniques include 1) dimpling and Ar-ion milling, or 2) focused Ga-ion beam (FIB) thinning. However, for samples that are made up of a collection of $100 \mathrm{~nm}-5 \mu \mathrm{m}$ diameter particles, this can be challenging. For example, preserving the arrangement of particles in an electrochemical experiment for ex situ microscopy is difficult. In addition, artifacts caused by redeposition and beam damage from ion beam removal techniques can be difficult to prevent. Here, we demonstrate the successful use of the ultramicrotome [1], usually used for soft and biological materials, for the preparation of thin inorganic particle samples.

We will show examples of how the ultramicrotome can prepare thin sections of inorganic powder samples used in energy storage applications. Li-ion battery electrodes generally consist of inorganic oxide particles mixed with an organic binder. By mapping the spatial distribution of ion state of charge in the electrodes particle-by-particle, it is possible to understand the mechanisms of the charge/discharge reaction and degradation in these materials [2-4]. In our first example, the ultramicrotome provides a way to make thin samples from battery electrodes in which the electrode particle arrangement is preserved and particles can be imaged with nanometer spatial resolution. Our second example will show how the ultramicrotome can be used to prepare thin sections of metallic Pd-alloy based powders for hydrogen storage. Surface modification of these powders can have a large impact on their thermal stability, hydrogen storage properties, and kinetics of hydrogen uptake and release [5]. The ultramicrotome provides a way to make samples such that we can image surface modifications that range from tens of nanometers to sub-nanometer dimensions.

\section{References}

[1] D. Studer and H. Gnaegi, Journal of Microscopy-Oxford 197 (2000)

[2] J.D. Sugar, et al., Journal of Power Sources 246 (2014)

[3] Y. Li, et al., Nat Mater 13 (2014)

[4] W.C. Chueh, et al., Nano Lett 13 (2013)

[5] P.J. Cappillino, et al., Langmuir 30 (2014)

[6] Sandia National Laboratories is a multi-program laboratory managed and operated by Sandia Corporation, a wholly owned subsidiary of Lockheed Martin Corporation, for the U.S. Department of Energy's National Nuclear Security Administration under contract DE-AC04-94AL85000. 


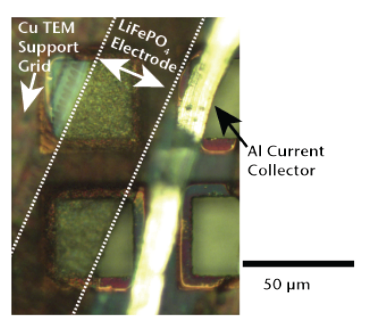

(a) $20 \mu \mathrm{m}$

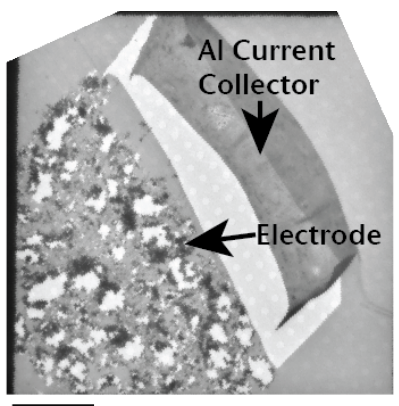

(b) $500 \mathrm{~nm}$

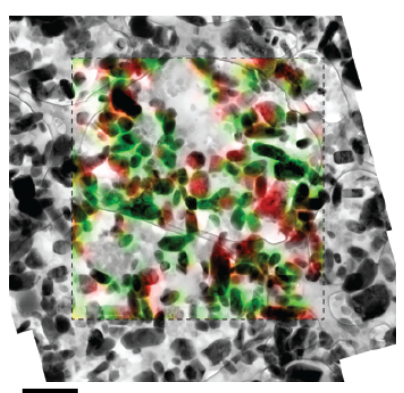

(c)

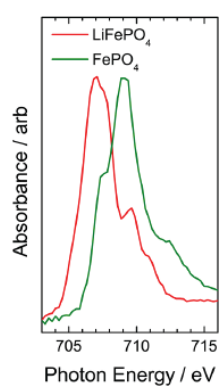

(d)

Figure 1: (a) Optical micrograph of $\mathrm{LiFePO}_{4}$ battery electrode sectioned with the ultramicrotome and attached to a TEM grid. The full section of the electrode is intact and the particle arrangement is preserved relative to the current collector. A lower magnification bright-field TEM image (b) shows that the cross section is electron transparent and a fraction of the particles have fallen off of the section. A STXM Fe state of charge map is overlaid a bright-field TEM image in (c) where the state of charge of the Fe cations is determined from the reference spectra shown in (d). It is possible to map the state of charge particle-by-particle in these Li-ion battery electrodes.

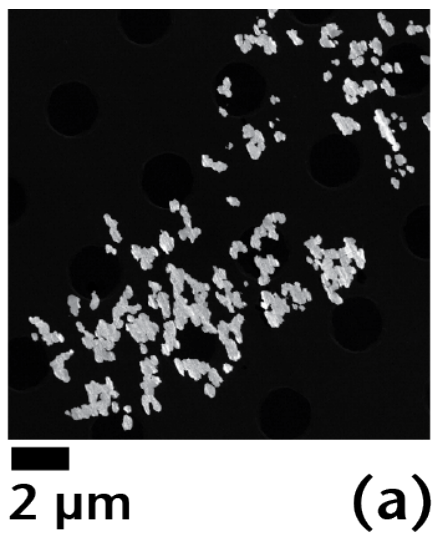

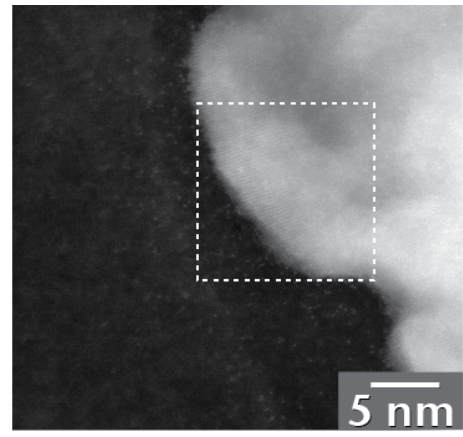

(b)

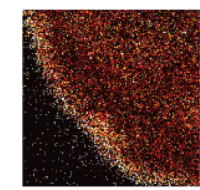

Rh at. fraction

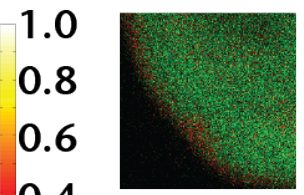

0.4

0.2 0

Pd map

Figure 2: (a) Lower magnification HAADF STEM image of Pd-alloy particles used for hydrogen storage. A higher magnification image near a particle surface is shown in (b) where lattice fringes are visible, demonstrating the high quality of this thin sample. Compositional analysis from EDS is shown in (c) where a $<1 \mathrm{~nm}$-thick surface modification layer enriched in $\mathrm{Rh}$ (by atomic layer electroless deposition) is visible. 